\title{
COMPARAÇÃO DE EQUAÇÕES DE CHUVAS INTENSAS PARA LOCALIDADES DO ESTADO DE SÃO PAULO
}

\section{PETERSON R. FIORIO ${ }^{1}$, SERGIO N. DUARTE ${ }^{2}$, GUILHERME DE O. RODRIGUES ${ }^{3}$, JARBAS H. DE MIRANDA ${ }^{4}$, RICHARD A. COOKE ${ }^{5}$}

RESUMO: Dada a importância das equações de chuvas intensas para o dimensionamento de obras de controle de enxurradas, este trabalho teve como objetivo comparar as alturas precipitadas geradas pelas equações de MARTINEZ \& MAGNI (1999) com aquelas obtidas com o programa PLUVIO 2.1, considerando as primeiras como padrão. Foram comparadas chuvas intensas de 10; 20; 30; 60; 120 e 1.440 minutos, e períodos de retorno de 2; 5; 10; 50 e 100 anos, para 30 localidades do Estado de São Paulo. Os resultados revelaram que, principalmente para as chuvas de 24 horas e período de retorno de 100 anos, houve desvios importantes para 4 postos localizados na região central e a leste do Estado. Para as demais localidades o programa apresentou bom desempenho.

PALAVRAS-CHAVE: relações intensidade-duração-frequência, precipitações, escoamento superficial.

\section{COMPARING RAINFALL INTENSITY DURATION RELATIONSHIPS FOR SITES OF THE STATE OF SÃO PAULO}

\begin{abstract}
Rainfall intensity durations relationships are extremely important in the design of systems for mitigating runoff losses. The objective of this work was to compare rainfall depths generated by the PLUVIO 2.1 software, with depths from the standard intensity duration curves developed by MARTINEZ \& MAGNI (1999). It was compared rainfall intensities of 10, 20, 30, 60, 120 and 1440 minute durations for 2, 5, 10, 50 and 100 year return periods for 30 sites in the state of São Paulo. The results showed that PLUVIO was effective, except in predicting the 24 hours rainfall from 100 year return period events in four locations in the central and eastern regions of the state.
\end{abstract}

KEYWORDS: intensity-duration-frequency relations, rainfall, surface runoff.

\section{INTRODUÇÃO}

As chuvas intensas são definidas como o conjunto de chuvas originadas de uma mesma perturbação meteorológica, cuja intensidade ultrapassa certo valor (chuva mínima). Essas perturbações podem variar de minutos até alguma dezena de horas (PRUSKI et al., 2006).

As equações de chuvas intensas são fundamentais para a estimativa do escoamento superficial, em situações onde não há dados medidos de vazão, ou quando a bacia hidrográfica está sofrendo processos de mudanças (MELO JÚNIOR et al., 2005; ENDO et al.,2005; OLIVEIRA et al., 2008). Por intermédio dos modelos chuva-vazão, é possível dimensionar uma série de obras hidráulicas, como drenos, bueiros, aduelas, pontilhões, obras contra cheias, e erosão hídrica, como

\footnotetext{
${ }^{1}$ Professor Doutor do Departamento de Engenharia de Biossistemas, ESALQ - USP.

${ }^{2}$ Prof. Associado 2 - Departamento de Engenharia de Biossistemas - ESALQ/USP Piracicaba - SP, snduarte@ usp.br.

${ }^{3}$ Graduando em Agronomia da ESALQ/USP, Casa do Estudante Universitário, Av. Pádua Dias 11 - ESALQ/USP, Piracicaba - SP, gorodrig@usp.br

${ }^{4}$ Prof. Associado 2, Depto. Engenharia de Biossistemas, Escola Superior de Agricultura "Luiz de Queiroz" - ESALQ/USP, jhmirand@usp.br.

${ }^{5}$ Instituição: Department of Agricultural and Biological Engineering (ABE), University of Illinois at Urbana/Champaign, Associate Professor of Soil and Water Engineering, rcooke@illinois.edu. 
os terraços agrícolas e os canais escoadouros, galerias de água pluviais, vertedores de barragens, entre outros (OLIVEIRA et al. 2005; PRUSKI et al., 2006; PEREIRA et al., 2007; ANA, 2009).

Existem várias fontes de dados de relações intensidade-duração-frequência para o Estado de São Paulo, com o destaque para o trabalho de MARTINEZ JÚNIOR \& MAGNI (1999), convênio do DAEE com a Escola Politécnica de São Paulo, que apresenta equações para 30 localidades do Estado, baseadas em séries, em geral superiores a 20 anos.

Essas expressões apresentam-se segundo a equação (1):

$$
i=A \cdot(t+B)^{C}+D \cdot(t+E)^{F} \cdot\left\{G+H \cdot \operatorname{Ln}\left[\operatorname{Ln}\left(\frac{T}{(T-1)}\right)\right]\right\}
$$

em que:

i - intensidade média da precipitação intensa, $\mathrm{mm}^{-1}{ }^{-1}$;

$\mathrm{t}$ - duração da precipitação, min;

$\mathrm{T}$ - período de retorno, anos; e

A, B, C, D, E, F, G, H - constantes de ajuste locais.

Uma segunda alternativa, que vem facilitando bastante os dimensionamentos, é o Programa PLUVIO 2.1, elaborado pelo Grupo de Pesquisas em Recursos Hídricos do Departamento de Engenharia Agrícola da Universidade Federal de Viçosa. Alguns trabalhos têm sido conduzidos visando à determinação de equações de chuvas intensas para diversas localidades do Brasil. Segundo FERREIRA et al. (2005) e PRUSKI et al. (2006), alguns pesquisadores determinaram elevado número de equações de chuvas intensas para localidades dos seguintes estados: Bahia, Espírito Santo, Goiás, Minas Gerais, Paraná, Rio de Janeiro, São Paulo e Tocantins. Os valores determinados para os parâmetros de todas estas equações estão disponíveis no banco de dados do PLÚVIO 2.1.

Para tal, foi desenvolvida uma metodologia para obtenção de equações de chuvas intensas em localidades em que essa não é conhecida, adaptada de PRUSKI et al. (1997), a qual está fundamentada no uso de um interpolador que permite obter os parâmetros da equação de intensidade-duração-frequência da precipitação a partir das informações disponíveis. Dessa forma, é possível quantificar os parâmetros da equação de intensidade-duração-frequência da precipitação para qualquer localidade desses estados e, consequentemente, obter a própria equação de chuvas intensas para essa área. Segundo PRUSKI et al. (2006), nessa interpolação, consideram-se apenas as informações inerentes às equações de chuvas intensas disponíveis, não sendo feita a análise do efeito da altitude do local e da presença de qualquer outro fator que possa ser condicionador da precipitação (presença de serras, represas, oceano, etc.), nos valores dos parâmetros de ajuste da equação de intensidade-duração-frequência da precipitação. A interpolação é realizada, independentemente, para cada um dos parâmetros desta equação (K, a, b, c), sendo utilizadas na interpolação todas as informações das localidades dos estados em que as equações são conhecidas. $\mathrm{O}$ fator de ponderação empregado, na consideração de cada localidade, corresponde ao inverso da quinta potência da distância entre as localidades em que os parâmetros são conhecidos e o local (latitude e longitude) para o qual a equação é pretendida.

Assim, tendo em vista a maior facilidade de se operar o Programa PLUVIO 2.1, e considerando o maior tamanho das séries de dados de MARTINEZ \& MAGNI (1999), e seu tradicional uso pelo DAEE-SP, este trabalho teve como objetivo comparar essas duas fontes de valores de precipitação intensa, tomando a segunda como padrão.

\section{MATERIAL E MÉTODOS}

\section{- Modelo Pluvio 2.1}

O software Pluvio 2.1 (Figura 1) espacializa os dados de chuvas intensas pelo método do inverso da quinta potência da distância, por ser esta uma das 28 combinações entre formas de 
interpolação que apresentaram melhores resultados na estimativa da intensidade máxima média de precipitação (CECÍLIO \& PRUSKI, 2003). Segundo esses autores, o erro médio percentual encontrado em testes foi igual a 19,37\%, valor considerado aceitável em se tratando de chuvas intensas.

Além disso, o uso da quinta potência da distância faz com que localidades mais próximas àquelas de interesse tenham maior peso na interpolação dos parâmetros da equação, uma vez que são consideradas todas as localidades em que a equação de chuvas intensas é conhecida nos Estados da Bahia, Espírito Santo, Minas Gerais, Paraná, Rio de Janeiro, São Paulo e Tocantins (PRUSKI et al., 2006).

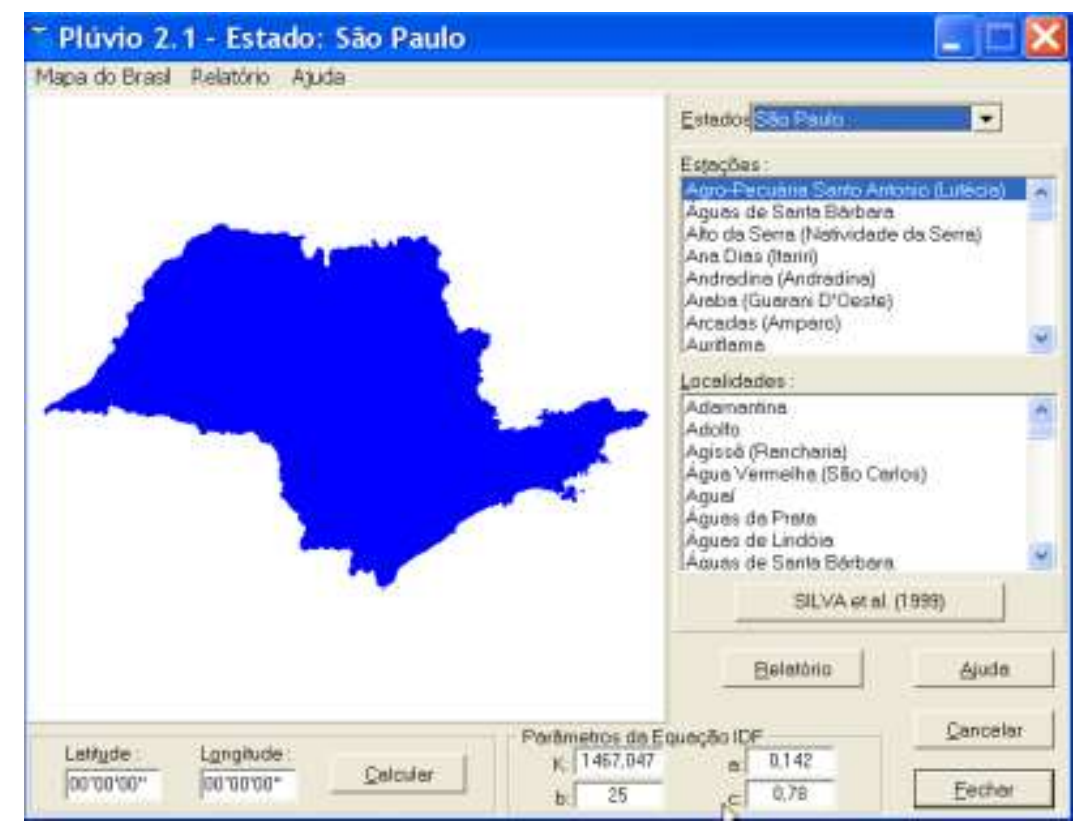

FIGURA 1. Tela do Programa Pluvio 2.1. Software screen of Pluvio 2.1 program.

Dessa forma, é permitido gerar a equação de chuva intensa para qualquer coordenada do Estado. Entretanto, os dados de base de chuvas do Programa PLUVIO (SILVA et al., 1999) não possuem séries tão longas quanto o trabalho de MARTINEZ \& MAGNI (1999).

As expressões do programa PLUVIO 2.1 seguem o modelo apresentado na equação (2):

$$
i=\frac{k T^{a}}{(t+b)^{c}}
$$

em que:

$\mathrm{i}$ - intensidade média da precipitação intensa, $\mathrm{mm} \mathrm{h}^{-1}$;

$\mathrm{t}$ - duração da precipitação, min;

$\mathrm{T}$ - período de retorno, anos; e

$\mathrm{k}, \mathrm{a}, \mathrm{b}, \mathrm{c}-$ constantes de ajuste locais.

\section{- Localidades do Estado de São Paulo}

Foram calculadas, pelas equações (1) e (2), citadas anteriormente, as alturas precipitadas para as 30 localidades apresentadas por MARTINEZ \& MAGNI (1999) (Figura 2), contemplando 6 durações e 5 períodos de retorno distintos, totalizando 30 precipitações para cada localidade. As durações foram de $10 ; 20 ; 30 ; 60 ; 120$ e 1.440 minutos; os períodos de retorno foram de $2 ; 5 ; 10 ; 50$ e 100 anos. 


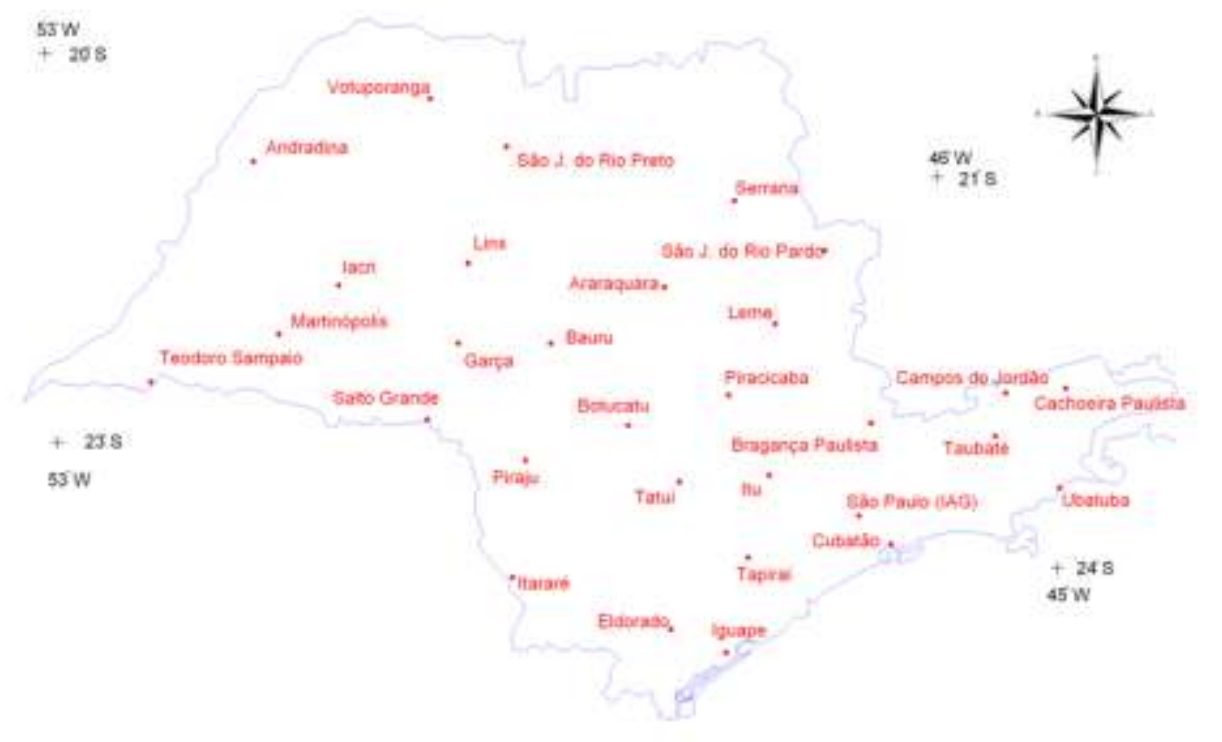

FIGURA 2. Localidades do Estado de São Paulo contempladas no estudo. Sites in São Paulo State used in this study.

As precipitações calculadas com as equações geradas pelo PLUVIO 2.1 foram comparadas com as de MARTINEZ \& MAGNI (1999) por meio de gráficos de dispersão, utilizando regressão linear, passando pela origem e coeficiente de determinação $\left(\mathrm{R}^{2}\right)$.

Com o objetivo de avaliar o desvio da reta de regressão em relação à reta $1: 1$, foi calculado o índice d de Willmott, para as 30 localidades, dado pela equação (3). Esse índice assume o valor máximo de 1,0 quando a reta de regressão coincide com a reta 1:1.

$$
\mathrm{d}=1-\left[\frac{\sum(\mathrm{Ei}-\mathrm{Pi})^{2}}{\sum(|\mathrm{Ei}-\mathrm{P}|+|\mathrm{Pi}-\mathrm{P}|)^{2}}\right]
$$

em que:

Ei - Valor estimado pelo PLUVIO 2.1;

Pi - Valor-padrão dado por MARTINEZ \& MAGNI (1999); e

P - média dos valores-padrão.

Foi calculado o índice de concordância c, proposto por ALLEN et al.(1998), como o produto do índice d de Willmott e o coeficiente de correlação R. Foram considerados aceitáveis os gráficos em que o PLUVIO 2.1 gerou dados cujo índice c foi maior que 0,85 , o que indica um desempenho ótimo, segundo ACOSTA et al.(2009).

\section{RESULTADOS E DISCUSSÃO}

$\mathrm{Na}$ Tabela 1, encontram-se listados os valores dos coeficientes locais interpolados das equações intensidade-duração-frequência do modelo utilizado no Programa PLUVIO 2.1 (Equação 2). As equações de base desse modelo foram ajustadas para cada localidade que dispunha de dados, utilizando o método da regressão múltipla linear, que é empregado após a linearização da Equação 2 . 
TABELA 1. Coeficientes das equações utilizadas pelo Programa PLUVIO 2.1, para diferentes localidades do Estado de São Paulo. Equations coeficients used by PLUVIO 2.1 software for different cities in the state of São Paulo.

\begin{tabular}{lcccc}
\hline Localidade & $\mathbf{K}$ & $\mathbf{a}$ & $\mathbf{b}$ & $\mathbf{C}$ \\
\hline Andradina & $1.844,723$ & 0,126 & 25,000 & 0,829 \\
Araraquara & $2.003,460$ & 0,125 & 25,016 & 0,841 \\
Bauru & 703,773 & 0,127 & 5,973 & 0,678 \\
Botucatu & $1.280,237$ & 0,113 & 24,999 & 0,718 \\
Bragança Paulista & $2.000,000$ & 0,151 & 19,787 & 0,904 \\
Cachoeira Paulista & $1.999,911$ & 0,157 & 25,000 & 0,801 \\
Campos do Jordão & $1.867,022$ & 0,127 & 20,838 & 0,896 \\
Cubatão & $1.114,037$ & 0,132 & 21,490 & 0,671 \\
Eldorado & $1.677,460$ & 0,131 & 25,000 & 0,799 \\
Garça & 577,565 & 0,148 & 5,027 & 0,611 \\
Iacri & $1.389,560$ & 0,126 & 24,962 & 0,742 \\
Iguape & 745,326 & 0,170 & 25,000 & 0,558 \\
Itararé & $1.439,516$ & 0,164 & 5,000 & 0,869 \\
Itu & $1.868,411$ & 0,121 & 24,996 & 0,827 \\
Leme & $1.424,017$ & 0,125 & 25,000 & 0,780 \\
Lins & $1.999,999$ & 0,108 & 21,015 & 0,860 \\
Martinópolis & $1.999,982$ & 0,136 & 24,661 & 0,856 \\
Piracicaba & $1.773,932$ & 0,173 & 24,999 & 0,798 \\
Piraju & $1.477,426$ & 0,134 & 24,939 & 0,749 \\
Salto Grande & $2.000,00$ & 0,132 & 21,831 & 0,880 \\
S. José do Rio Pardo & 701,377 & 0,119 & 11,497 & 0,642 \\
S. José do Rio Preto & $1.669,793$ & 0,109 & 25,000 & 0,792 \\
São Paulo & $1.999,992$ & 0,108 & 20,206 & 0,838 \\
Serrana & $1.999,955$ & 0,112 & 25,000 & 0,836 \\
Tapiraí & 940,296 & 0,118 & 13,848 & 0,739 \\
Tatuí & 891,227 & 0,119 & 8,397 & 0,672 \\
Taubaté & 913,707 & 0,158 & 5,000 & 0,772 \\
Teodoro Sampaio & $1.856,792$ & 0,129 & 24,999 & 0,828 \\
Ubatuba & 675,631 & 0,151 & 17,851 & 0,635 \\
Votuporanga & $1.999,489$ & 0,146 & 24,950 & 0,835 \\
\hline
\end{tabular}

$\mathrm{Na}$ Tabela 2, são apresentados os valores de coeficiente angular da reta de regressão e do coeficiente de determinação para as 30 localidades contempladas. Observando-se a Tabela 2, verifica-se que das 30 localidades analisadas apenas 4 se encontram fora da faixa de adequação estabelecida ( c < 0,85), sendo as 4 por superestimativa das precipitações. Na Figura 3, é apresentada a localização dessas estações. Pode-se observar que as mesmas estão localizadas no centro e a leste do Estado de São Paulo. 
TABELA 2. Localidades analisadas, coeficiente angular da reta de regressão (a), índice de Willmott (d), índice de ALLEN et al. (1998) (c) e coeficiente de determinação $\left(\mathrm{R}^{2}\right)$. Sites analyzed, linear regression coefficient (a), Wilmott index (d), ALLEN (1998) index (c) and coefficient of determination $\left(\mathbf{R}^{2}\right)$.

\begin{tabular}{|c|c|c|c|c|}
\hline Localidade & $\mathbf{a}$ & d & c & $\mathbf{R}^{2}$ \\
\hline Andradina & 1,0662 & 0,9876 & 0,9708 & 0,9663 \\
\hline Araraquara & 1,0992 & 0,9704 & 0,9375 & 0,9333 \\
\hline Bauru & 1,0946 & 0,9617 & 0,9115 & 0,8984 \\
\hline Botucatu & 1,4800 & 0,8589 & $0,8158 *$ & 0,9022 \\
\hline Bragança Paulista & 0,9704 & 0,9932 & 0,9799 & 0,9734 \\
\hline Cachoeira Paulista & 1,4465 & 0,8392 & $0,7025^{*}$ & 0,8695 \\
\hline Campos do Jordão & 0,8047 & 0,9346 & 0,8645 & 0,8557 \\
\hline Cubatão & 1,0196 & 0,9885 & 0,9676 & 0,9582 \\
\hline Eldorado & 1,0846 & 0,9665 & 0,9201 & 0,9062 \\
\hline Garça & 1,5249 & 0,7592 & $0,6531 *$ & 0,7401 \\
\hline Iacri & 1,1347 & 0,9748 & 0,9517 & 0,9532 \\
\hline Iguape & 1,0959 & 0,9697 & 0,9272 & 0,9142 \\
\hline Itararé & 0,8658 & 0,9581 & 0,9272 & 0,9145 \\
\hline Itu & 1,1560 & 0,9617 & 0,9316 & 0,9383 \\
\hline Leme & 1,0280 & 0,9746 & 0,9325 & 0,9154 \\
\hline Lins & 0,8204 & 0,9525 & 0,9218 & 0,9366 \\
\hline Martinópolis & 0,9713 & 0,9770 & 0,9545 & 0,9545 \\
\hline Piracicaba & 1,1810 & 0,9555 & 0,9221 & 0,9313 \\
\hline Piraju & 1,1056 & 0,9910 & 0,9879 & 0,9937 \\
\hline Salto Grande & 0,9960 & 0,9948 & 0,9837 & 0,9779 \\
\hline S. José do Rio Pardo & 1,5239 & 0,8472 & $0,8001 *$ & 0,8921 \\
\hline S. José do Rio Preto & 1,1817 & 0,9315 & 0,8729 & 0,8781 \\
\hline São Paulo & 1,1518 & 0,9504 & 0,8729 & 0,9044 \\
\hline Serrana & 1,0442 & 0,9780 & 0,9426 & 0,9290 \\
\hline Tapiraí & 0,9695 & 0,9871 & 0,9632 & 0,9521 \\
\hline Tatuí & 1,3737 & 0,9179 & 0,8933 & 0,9472 \\
\hline Taubaté & 1,0151 & 0,9780 & 0,9386 & 0,9211 \\
\hline Teodoro Sampaio & 1,0883 & 0,9848 & 0,9695 & 0,9692 \\
\hline Ubatuba & 0,8494 & 0,9804 & 0,9677 & 0,9744 \\
\hline Votuporanga & 1,1820 & 0,9658 & 0,9506 & 0,9687 \\
\hline
\end{tabular}

* = índice c de ALLEN et al. (1998) menor que 0,85

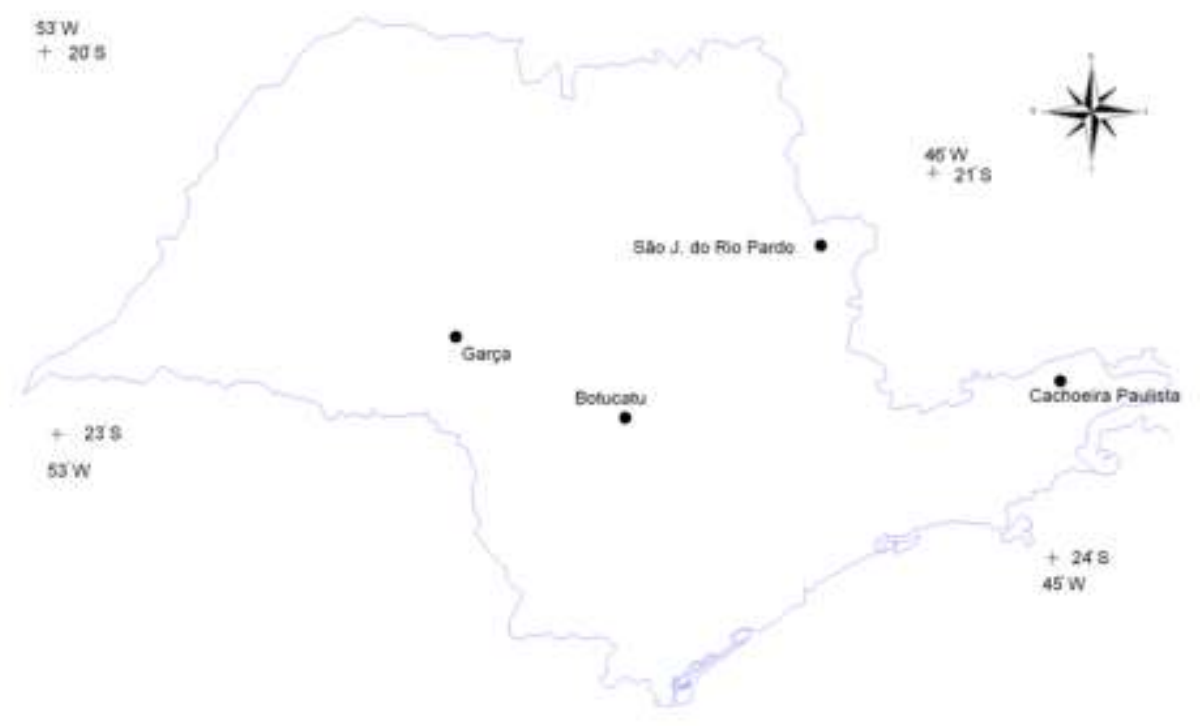

FIGURA 3. Localidades do Estado de São Paulo onde não houve bom desempenho do Programa PLUVIO 2.1. Sites in the state of São Paulo where PLUVIO 2.1 did not perform well. 


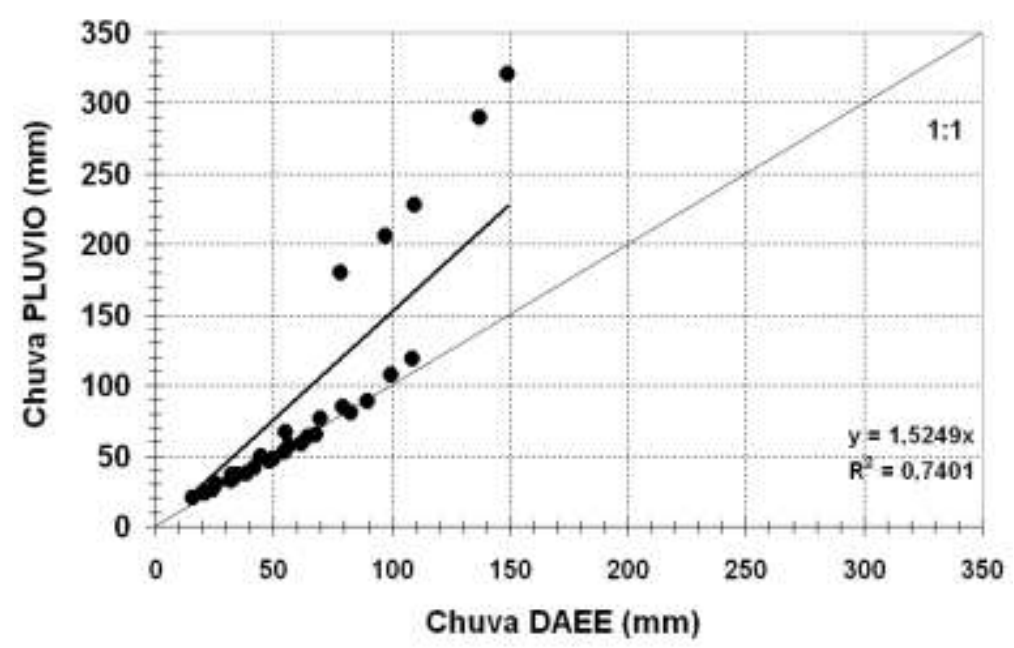

(A)

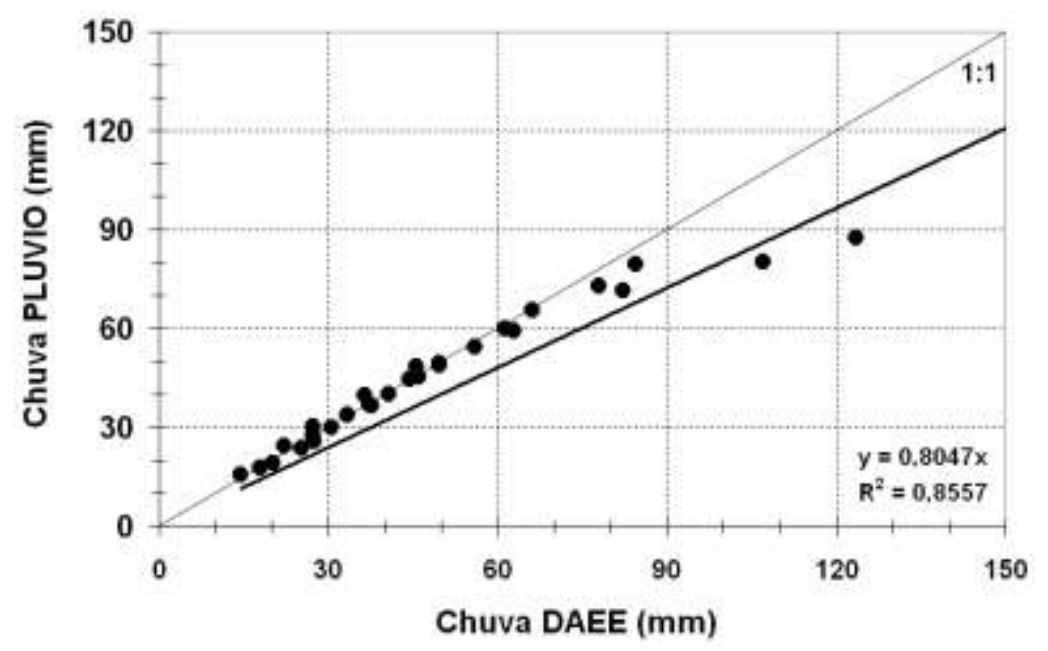

(B)

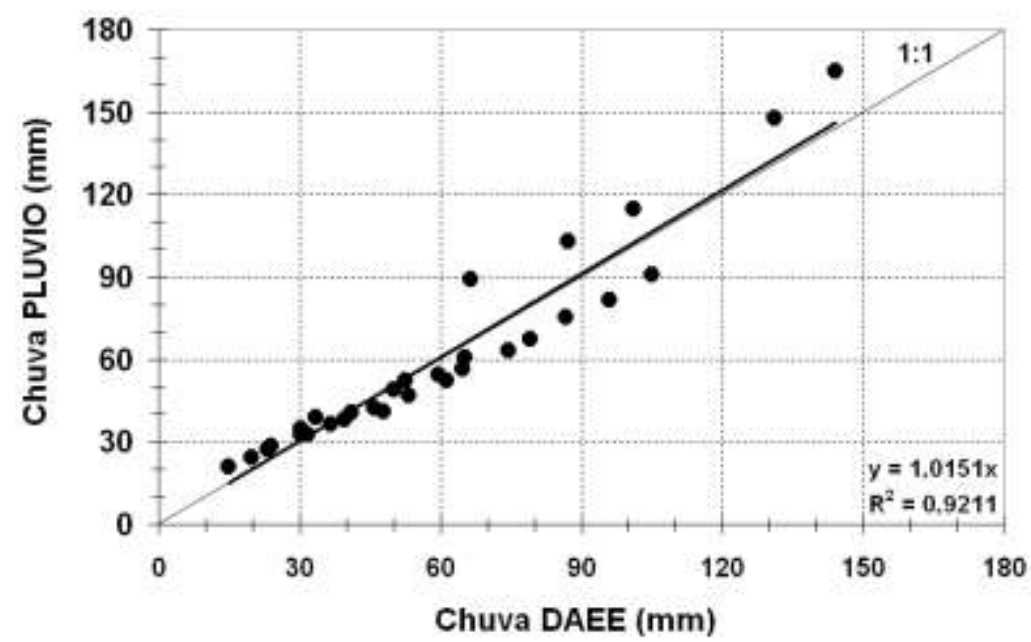

(C)

FIGURA 4. Gráficos de dispersão entre as precipitações calculadas pela equação do DAEE (MARTINEZ e MAGNI, 1999) e as calculadas com o programa PLUVIO 2.1. Em (A) a maior superestimativa (Cidade de Garça); em (B) a maior subestimativa (Cidade de Campos do Jordão) e em (C) o coeficiente angular mais próximo da unidade (Cidade de Taubaté). Dispersion graphs between rainfall calculated by the PLUVIO 2.1 software and the DAEE equations (MARTINEZ e MAGNI, 1999). In (A) the location in which rainfall was most overestimated (Garça City), (B) the location in which rainfall was most underestimated (Campos do Jordão City) and (C) the location where the results were most closely matched (Taubaté City). 
Por meio da Figura 4a , pode-se verificar a comparação das chuvas para a estação de Garça, onde ocorreu a maior superestimativa $(52,49 \%)$. Pode-se notar que os maiores desvios se deram para as maiores alturas precipitadas, ou seja, as chuvas de 1.440 minutos. Isto significa que o uso do PLUVIO 2.1 para o dimensionamento de estruturas cuja área de contribuição tenha um tempo de concentração pequeno não é muito problemático. Enquadram-se nesse caso os terraços em desnível e várias obras de drenagem urbana, como as galerias de água pluvial de ruas e avenidas (PINTO et al., 1996). Já para estruturas com área de captação grande, como vertedores de barragens de porte médio, bueiros e outras travessias, o uso do PLUVIO 2.1, para essas localidades, pode encarecer as obras. O mesmo ocorre para o cálculo do espaçamento entre drenos agrícolas e das seções de terraços em nível, em que se utilizam, geralmente, chuvas de 6 e 8 horas de duração, respectivamente.

Uma alternativa para a correção desse problema seria a adoção de duas ou mais equações para uma mesma localidade, cada uma englobando uma faixa de duração e período de retorno, tal como sugerem COSTA \& PRADO (2003). Entretanto, haveria que se ponderar para o fato de esse procedimento causar dificuldades na espacialização dos dados.

$\mathrm{Na}$ Figura 4B, é apresentada a correlação para a localidade onde ocorreu a maior subestimativa (19,53\%), ou seja, a localidade de Campos do Jordão. Verifica-se que também para essa localidade os maiores desvios se deram para as chuvas de 1.440 minutos.

Na Figura 4C, pode-se observar a correlação que apresentou o melhor ajuste ocorrido, que foi obtido para a localidade de Taubaté. Nesse caso, a reta 1:1 praticamente coincide com a reta de regressão, garantindo segurança para os dimensionamentos a serem realizados nesse município.

\section{CONCLUSÕES}

O programa PLUVIO 2.1 pode ser utilizado para o Estado de São Paulo, com exceção de quatro localidades pertencentes às regiões central e leste, onde existem desvios para as precipitações geradas, principalmente para as de duração de 24 horas e período de retorno de 100 anos. Nessas regiões, o programa superestima as precipitações, quando comparadas às de MARTINEZ E MAGNI (1999).

\section{AGRADECIMENTOS}

Os autores agradecem ao Ministério da Ciência e Tecnologia (MCT), ao Conselho Nacional de Desenvolvimento Científico e Tecnológico $(\mathrm{CNPq})$ e à Fundação de Amparo à Pesquisa do Estado de São Paulo (FAPESP), pelo apoio financeiro a esta pesquisa, através do Instituto Nacional de Ciência e Tecnologia em Engenharia da Irrigação (INCTEI).

\section{REFERÊNCIAS}

ACOSTA, J.F.; CARDOZO, N.P.; MARIN, F.R. Avaliação do desempenho de um modelo de estimativa de saldo de radiação em algumas culturas. In: CONGRESSO BRASILEIRO DE AGROMETEOROLOGIA, 16., 2009, Belo Horizonte. CD-ROM.

ALLEN, R.G.; PEREIRA, L.S.; RAES, D.; SMITH, M. Crop evapotranspiration: guidelines for computing crop water requirements. Rome: FAO, 1998. 300p. (FAO Irrigation and Drainage Paper, 56)

ANA. Agência Nacional das Águas. Hidro web: sistemas de informações hidrológicas. Disponível em http://hidroweb.ana.gov.br. Acesso em: 1dez. 2009.

CECÍLIO, R. A.; PRUSKI, F. F. Interpolação dos parâmetros da equação de chuvas intensas com uso do inverso de potências da distância. Revista Brasileira de Engenharia Agrícola e Ambiental, Campina Grande, v.7, n.3, p.501-504, 2003. 
COSTA, A. R. da; PRADO, L. A. Espacialização de chuvas intensas para o Estado de Goiás e o sul de Tocantins. Revista Engenharia Agrícola, Jaboticabal, v.23, n.2, p.268-273, 2003.

ENDO, C.K.; SAMPAIO, S.C.; GOMES, B.M.; SILVESTRO, M.G.; FRIGO, E.P.; CAOVILLA, F.A. Análise de consistência de dados de precipitação da região oeste do Estado do Paraná. In: CONGRESSO BRASILEIRO DE ENGENHARIA AGRÍCOLA, 34., 2005, Canoas. Anais... CDROM.

FERREIRA, R.C.; GONÇALVES, A.C.A.; CARAMORI, P.H.; FREITAS, P.S.L.; BERTONHA, A.; WREGE, M.S. Variabilidade espacial da precipitação para os meses de outono e inverno no Estado do Paraná. In: CONGRESSO BRASILEIRO DE ENGENHARIA AGRÍCOLA, 34., 2005, Canoas. Anais... CD-ROM.

MARTINEZ JUNIOR, F.; MAGNI, N.L.G. Equações de chuvas intensas do Estado de São Paulo. São Paulo: Departamento de Águas e Energia Elétrica, Escola Politécnica da Universidade de São Paulo, São Paulo. 1999. 125 p.

MELO JUNIOR, J.C.F.; SOUSA, E.F.; BERNARDO, S. Análise espacial do regime de chuvas na região hidrográfica costeira do Sudeste brasileiro. In: CONGRESSO BRASILEIRO DE ENGENHARIA AGRÍCOLA, 34., 2005, Canoas. Anais... CD-ROM.

OLIVEIRA, L.F.C. et al. Intensidade-duração-frequência de chuvas para localidades no Estado de Goiás e Distrito Federal. Pesquisa Agropecuária Tropical, Goiânia, n.35, p.13-18, 2005.

OLIVEIRA, L.F.C.; ANTONINI, J.C.A.; GRIEBELER, N.P. Estimativa de chuvas intensas para o Estado de Goiás. Engenharia Agrícola, Jaboticabal, v.28, n.1. p.22-33, 2008.

PEREIRA, C.E.; SILVEIRA, A.; SILVINO, A.N.O. Estudo de chuvas intensas e estimativa da equação IDF para a cidade de Barra dos Bugres - MT. In: CONGRESSO DA ASSOCIAÇÃO BRASILEIRA DE RECURSOS HÍDRICOS, 17., 2007, São Paulo. Anais... CD-ROM.

PINTO, F.A.; FERREIRA, P.A.; PRUSKI, F.F.; ALVES, A.R.; CECON, P.R. Equações de chuvas intensas para algumas localidades do Estado de Minas Gerais. Engenharia Agrícola, Jaboticabal, v.16, n.1, p.91-104, 1996.

PRUSKI, F.F.; CALIJURI, M.L.; BHERING, E.M.; SILVA, J.M.A. Metodologia baseada no uso de sistemas de informações geográficas para obtenção de equações de chuvas intensas em qualquer localidade do Estado do Paraná. Engenharia na Agricultura, Viçosa, v.5, n.3, p.254-265, 1997.

PRUSKI, F.F.; SILVA, D.D.; TEIXEIRA, A.F.; CECÍLIO, R.A.; SILVA, J.M.A.; GRIEBELER, N.P. Hidros. Dimensionamento de sistemas hidroagrícolas. Viçosa: Editora UFV. 2006. 259 p.

SILVA, D.D. et al. Estimativa e espacialização dos parâmetros da equação de intensidade-duração-frequência para o Estado de São Paulo. Engenharia na Agricultura, Viçosa, v.7, n.2, p.70-87, 1999. 